\title{
Preliminary Experience with Combined Therapy using Rifampicin and Isoprodian (L73A)
}

\author{
E. FREERKSEN \\ Forschungsinstitut Borstel, 2061 Borstel, West Germany
}

We should like to present to you some findings in leprosy patients which were not obtained by conventional trials, but by the results of treatment with the same combined therapy as used at different centres and consisting of rifampicin + Isoprodian. We did not have the intention of comparing this form of medication with others; we wanted to help several groups of dangerously ill patients by giving them a highly effective treatment.

Our conviction that the above-mentioned treatment represents the most effective therapy today, is based on our experimental results and on some clinical random trials carried out earlier. I know very well that in so doing I violate an old habit; this is why I have briefly to substantiate this opinion.

What significance can trials still have today? Prior to the early nineteen-forties there did in fact not exist any effective chemotherapy. At this moment in history leprologists encountered the sulphones, i.e. a real chemotherapeutic agent, for the first time, and it became possible to assess a drug against the background "Nil". Only under such conditions could trials in the classical sense be undertaken. Today we have at our disposal several effective substances. Therefore we no longer need to decide between "activity" and "inactivity", but rather have to distinguish degrees of efficiency by comparison.

This becomes more difficult and complicated from the technical point of view, when the differences in activity are small. For this reason we may use only those patients who can furnish any useful criteria for a comparative study. This is why, above all, lepromatous cases or at least bacteriologically positive ones are suitable for such trials, since the decrease in the number of bacteria provides the most important criterion for the assessment of anti-bacterial activity. Starting from the principle that all antileprosy drugs available today are active because of their inhibition of bacterial multiplication or killing power, we have to concede that no drug now available will accelerate the rate of clearance of bacteria from the tissues. The period of time taken for this to happen, could be regarded as a parameter. The morphological changes in the bacteria and their quantitative estimation (MI) are often in use. But our knowledge in this field is scantier than often believed.

(1) Trials in the form used in the past are to be justified not only in respect of the bacteriological criteria, but also regarding the choice of the patients. In order to satisfy the minimum requirements for statistical interpretation, we need at 
least 3 groups if only one new substance is to be assessed, i.e. (a) 1 untreated control group, (b) 1 group treated with a substance of known activity and (c) a third group treated with the substance to be tested. For statistical reasons, each of these 3 groups should consist of at least 30 cases who must be strictly comparable in all essential respects. That means about 100 cases per trial carried out in the most simple manner. I have seen many leprosy patients and leprosaria, but up to now I have not found 100 identical cases in the same hospital, who could be used for such a trial. That is why I hold the opinion that scientifically acceptable trials are no longer possible. Trials with groups consisting of 4 or 8 or even 20 cases, observed only over a period of a few months are thus of no scientific value and do not furnish sufficient information because only slight (but practically essential) differences in effectiveness will be compared, even with highly effective forms of medication.

Although for technical and quantitative reasons true scientific trials are impossible, we should seriously question ourselves, whether such trials could at all be justified. Could we as doctors allow-just for the sake of scientific reasons-that one group of patients remain untreated, although they need treatment?

(2) The demand that only patients who have not had previous treatment should be taken into such trials is based on a reasonable foundation. But how to find 100 such cases, who have not even had dapsone? In the leprosaria I visited I have hardly come across any untreated patient with lepromatous leprosy; we are always dealing with patients who have had treatment somehow and at sometime or other except for those few fresh cases who often do not fit into the trial protocol. This demand for non-treated cases is theoretically correct, but not attainable in practice.

(3) All our patients we shall show-with a few exceptions-have had previous treatment, the results of which in each individual patient serve as control data. At the outset of combined therapy, our cases were bacteriologically positive and the clear majority lepromatous. Nearly all of them were previously treated with dapsone, not only for 6 or 12 months but often for a period of 6,12 or even 20 years. At the beginning of our therapy all these cases were still highly positive. The previous treatment had been ineffective.

(4) During this colloquium we should clearly define the decisive criteria for the effectiveness of a prescribed treatment. In treating almost identical cases receiving $25 \mathrm{mg}$ of dapsone or $100 \mathrm{mg}$ dapsone or rifampicin or even rifampicin in combination you will probably not notice any bacteriological differences at all during the first 3 or even 6 months-and if any do exist, they can be irrelevant for statistical purposes or difficult to standardize.

The actual and decisive criterion determining the value of a therapy in the case of infectious diseases such as leprosy and all the other mycobacterioses (including tuberculosis) is the absence of relapse. We cannot alter the period of time necessary for this proof. One year remains 1 year and 5 years remain 5 years; today we simply should not judge definitely the value of different forms of therapy showing relatively similar initial effectiveness. That goes naturally for the form of treatment reported here.

(5) The absence of relapses can only be observed and investigated, if we are courageous enough to stop treatment at a given point of time. This is permissible only if the patients can remain under observation for a long time after withdrawl of treatment. This is also only possible under special and rare circumstances and 
where therapy can be started should relapses occur. The point of time, chosen arbitrarily, but not without foundation, for stopping treatment arrives when 6 successive monthly bacteriological tests are negative.

(6) As mentioned above, we think that too great a reliance on the morphological changes of the bacteria (MI) as a criterion for the effectivity of a therapy is open to question. We know that nearly all species of mycobacteria investigated for this purpose, reveal the ability to adapt to changing environmental conditions, first of all by granulation, fragmentation, etc. Chemotherapeutic agents themselves represent an environmental influence on the mycobacteria in (living) media. On the other hand (especially under insufficient treatment) the recurrence of all bacterial forms can be possible. The best criterion of a therapeutic effect is tissue clearance demonstrated repeatedly. That is why the decisive criterion is not the MI, but the reduction to zero of the BI. All our investigations were carried out in accordance with the concept I explained in detail in September, 1972 (Freerksen, E. and M. Rosenfeld: Fundamental data, methods and goals of present research on the treatment of leprosy. $Z$. Tropenmedizin 24 (1973), 17-25). 